\title{
Medical Toxicologist Attitudes on Compensation for Services Provided to Poison Control Centers
}

\author{
Steven R. Offerman • Jeffrey Brent • Paul M. Wax
}

Published online: 25 March 2010

(C) American College of Medical Toxicology 2010

To the Editor,

Little is known regarding medical toxicologists' financial compensation by poison control centers (PCCs) in the United States or about toxicologist attitudes regarding compensation. Our aim was to survey and describe the opinions of American College of Medical Toxicology (ACMT) member toxicologists regarding their compensation by PCCs.

The ACMT practice committee conducted an anonymous survey of all ACMT members. Three times between July and September 2008, ACMT members were contacted by e-mail and asked to fill out an online survey. An additional attempt was made to collect survey responses at the ACMT national meeting. No compensation was provided for study participation. The survey format was designed to be short and simple in order to maximize response rates.

One hundred fifty-two ACMT members provided survey responses (27.6\%). Forty (26.2\%) respondents described themselves as poison center directors, $24(15.8 \%)$ as associate/assistant directors, $51(33.6 \%)$ as poison center

\author{
S. R. Offerman \\ California Poison Control System, \\ Department of Emergency Medicine, \\ Kaiser Permanente South Sacramento Medical Center, \\ Sacramento, CA, USA \\ J. Brent $(\bowtie)$ \\ Toxicology Associates, \\ University of Colorado Health Sciences Center, \\ Denver, CO, USA \\ e-mail: jeffrey.brent@ucdenver.edu \\ P. M. Wax \\ Department of Emergency Medicine, \\ UT Southwestern Medical Center, \\ Dallas, TX, USA
}

consultants, and 17 (\%) as providing teaching/administrative services. Twenty (13.2\%) respondents reported having no poison center involvement. Of those respondents without PCC involvement, 14 (70\%) reported being geographically distant from their PCC.

All respondents felt that toxicology consultations provided by medical toxicologists were useful $(100 \%)$. One hundred twenty-seven (84.1\%) strongly agreed and 24 agreed $(15.9 \%)$ with this statement. One hundred fortyeight (97.4\%) agreed that medical toxicologists should be compensated for their time. Only two respondents $(1.3 \%)$ disagreed.

One hundred six respondents $(70.2 \%)$ disagreed with the statement that toxicologists are fairly compensated for providing services to poison centers. Thirty-seven (24.5\%) of those "strongly disagreed." Only 34 respondents $(22.5 \%)$ felt that toxicologists are fairly compensated for their work.

Sixty-three (48.5\%) respondents reported "always" being compensated for their work at poison centers, whereas 36 $(27.7 \%)$ reported that they "never" were. Of those identified as PCC directors, $31(77.5 \%)$ reported always being compensated for their work. Only two (5\%) reported never being compensated. However, only $16(40 \%)$ of PCC directors felt that they were fairly compensated. Of nonPCC directors, only 20 (29.9\%) reported that they are always compensated for their work. Thirty-three $(49.3 \%)$ of nonPCC directors reported never getting compensation. Only 13 $(18.8 \%)$ felt that toxicologists are fairly compensated.

Our survey is limited by its low response rate, which limits our ability to extrapolate our findings to all ACMT member toxicologists. Some of the low response rate that we experienced may be related to ACMT members who do not provide services to a PCC or those receiving no compensation for their work. These particular members may not have responded because they felt the survey did 
not apply to them. Only $13.2 \%$ of respondents reported having no PCC involvement which, we feel, is likely much lower than that of the ACMT membership. Similarly, the number of ACMT toxicologists receiving no compensation for PCC services is likely to be higher than the $27.3 \%$ that we found.

The ACMT member toxicologists surveyed agree that they provide useful services and most feel that they should be compensated for their work. The majority, however, do not feel they are compensated fairly. Less than half of these toxicologists reported being fully compensated (47.7\%) and a large percentage $(27.3 \%)$ reported never receiving compensation for their PCC work.
It should be noted that respondents that identified themselves as PCC directors or assistant/associate directors are fully compensated more frequently than non-PCC directors. Predictably, these PCC directors also feel that toxicologist compensation is fair more often than non-PCC directors.

It is clear that, while PCCs frequently rely on medical toxicologists to provide services, these physicians often go unpaid. While this lack of compensation for specialty services may be common in medical toxicology, it would clearly be unacceptable in other specialties. Our findings suggest that ACMT members feel that lack of appropriate compensation for PCC services is a widespread problem and thus future college activities should address this issue. 